\title{
Application and Research of Integrated Model Between Substation and Dispatch Center
}

\author{
Ning Jiang ${ }^{1}$, Lin $\mathrm{Li}^{1}$, Ning Kang ${ }^{1}$, Guodong $\mathrm{Li}^{1}$, Lingxu Guo ${ }^{2}$ and Jian Chen ${ }^{2}$ \\ ${ }^{1}$ Tianjin Electric Power Research Institute, State Grid, Tianjin, China \\ ${ }^{2}$ Tianjin Electric Dispatch and Control Center, State Grid, Tianjin, China
}

\begin{abstract}
This paper realizes cooperative modeling between intelligent substation and dispatch center based on IEC 61850 standard. The functions of the System Configuration Utility of Substation Automation System is regulated; The equipment and signal description in SCD file is regulated and expanded. The measuring model in CIM/E file is expend. The modeling mapping rules between IEC 61850 and IEC 61970 standard is developed. Classify the information referred the Information Specification of Protection Relay and the Information Specification of surveillance system. Introduce a project application case based on cooperative modeling method between substation and dispatch center. Introduce the modeling process and realization of engineering application. This paper realizes source maintenance and coordination sharing of the dispatch center and substation model and establishes integrated operation control center and substation maintenance system to meet the needs of safe and stable operation of the grid.
\end{abstract}

Keywords-integrated model; IEC 61850; source maintenance

\section{INTRODUCTION}

With the increasing pace of substation construction, put forward more rigorous requirements of the substation model management. Lack of a unified engineering implementation of technical standards, substation and dispatch center build model respectively, which exists problems such as non-standard naming, the monitoring signal names not standardized, no precise definition of description field in the SCD file of substation. It researches integrated model based on substation and dispatch center, realizing conversion from the IEC 61850 model directly into the dispatch center of the IEC 61970 model. It improves the interoperability of substation and dispatch center, to solve a series of problems caused by the model is not complete [1][2].

Currently, there are two kinds of standard model of the substation, which are SCD files and CIM files. These two models will be common in a long time [3]. SCD file focuses on the secondary equipment description, and the description of the primary equipment is not completely missing equipment parameters and other properties. CIM file focuses on the primary equipment description, and the description of the secondary equipment is missing [4]. It points out that the key differences between the two models are different description mechanism, the different object identification method, the different measurement and the state information expression, and the different class object attribute of the unified power system object in [5]. SCD file and CIM file are converted, and conversion method from model to graphics is researched in [69].

\section{MODELING METHOD}

\section{A. General Principle}

In accordance with the IEC61850 standard extension principle, the regulation of modeling requirements proposed by the dispatch center, regulate the primary and secondary equipment models, as well as association between equipment and signals. Compliant SCD file generated by substation export by conversion or directly from the system. CIM / E files provide substation model, and CIM / G files provide substation graphics. The dispatch center receives CIM / E files from substation, then complete the model without disturbance into the dispatch center using the combination model based CIM / E and other tools. Dispatch center receives CIM / G files from substation. After conversion from critical information, CIM / G files import to dispatch center with sharing the substation graphics. At the same time, independent conversion tool of cross-platform achieves the convert from SCD files to CIM / E files. Collaborative modeling methods described below.

\section{B. Functional Requirements}

The modeling regulates configuration tool function of the substation automation system, and regulates device and signal description in the SCD files, including measure models in the CIM / E files expanded to. The modeling formulates the development of mapping rules of IEC 61850 and IEC 61970 models. With reference to requirements of following electrical protection specification information and monitoring system specification information, information is managed classified. Using the system configuration tool, CIM / E files required by the dispatch center are derived from substation automatically, then checked. Through offline or online manner, CIM / E model files are used by the dispatch center, and provide model stitching and storage processing further.

Configuration tools of substation automation system should have the following features:

Firstly, substation secondary equipment functional model should be maintained. It should have the required features for IEC 61850-6 requirements, including IED Capability Description(ICD) files importing and using, the SCD files importing/exporting, and maintenance; 
Secondly, substation primary equipment functional model should be maintained. According to difference from sources and forms of information, it provides the appropriate means in order to maximize the use of existing resources. The first case is that System Specification Description(SSD) file is available, then use imported SSD file mode. The second case is that Scalable vector graphics(SVG) file from the existing substation monitoring system or CIM / G Language (CIM / G) graphics file is used to import SVG or CIM / G and provides functions based on SVG or CIM / G grid topological model. The third case is that as the initial source of information system configuration tools provide graphical editing functions, drawing substation wiring diagram, and building a network topology model.

Thirdly, associate primary or secondary equipment and signal model.

Fourthly, file checksum. File types include files described by Substation Configuration Language (SCL), SVG files, $\mathrm{CIM} / \mathrm{G}$ files, CIM/E files. Its features include syntax and semantics checking.

Fifthly, CIM/E files export. It implements model transformation in accordance with the mapping rules from the established IEC61850 model to IEC 61970 model, and exports the files.

\section{Model Extension}

Description specifications and expand of signal and equipment in SCD file is shown as follows:

1) Fill description in the section of desc in all levels of the model SCD. The description is described as an information source of source maintenance and unified whole network. The length of desc name is no longer than 64 bytes;

2) A description of the substation equipment maintains consistent with the dispatch center. It is named for the various types of equipment according to "DL / T 1171 Power grid equipment common model naming specification". The name is described in desc section of the corresponding model, which is full name containing the path. The full path name of device is the uniquely identify within the system.

3) A description of the various types of signals is mainly from desc description of Logic Node (LD) following all levels of functional model in secondary equipment. It uses natural names. Protective relaying and automatic safety devices are described as protected devices. Automation equipment is described according to the scope of the monitoring.

4) Data communication gateway machine (remote host) summarizes information of all the Intelligent Electronic Device(IED) in the bay level. It should be modeled as a separate IED. ExtractsLD related to the dispatch center. Reorganizes exchanging data, and develop data set.

5) Expand to associated portion between the signal and primary or secondary equipment. It only associated to logical node( $\mathrm{LN})$ expands to associated to Data Object(DO), which is an increase doName and fc portion in $<$ LNode>, e.g. doName $=$ "A.phsA", fc = "MX".

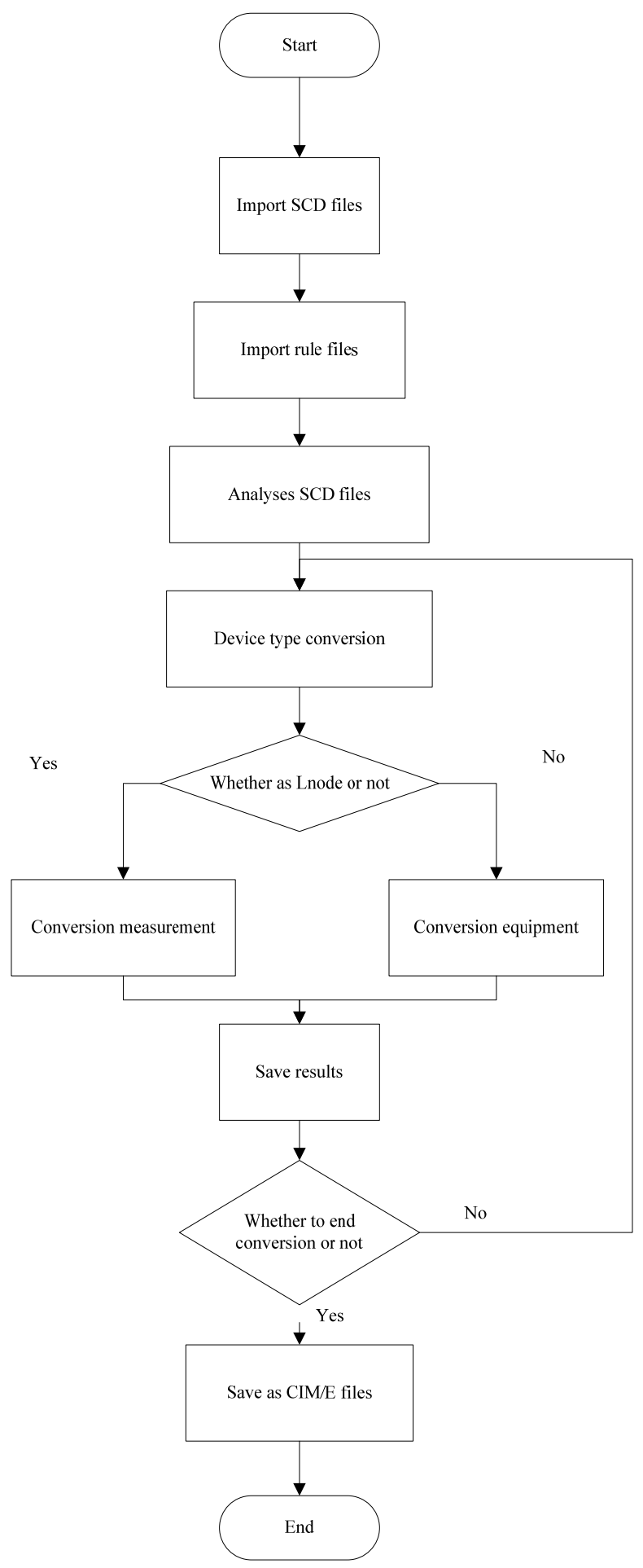

FIGURE I. SCD AND CIM/E FILE CONVERSION PROCESSING FLOW

On the basis of "CIM / E grid physical model description and exchange specification ", measurement model in the CIM / E file is expanded as following: 1) Analog and Discrete increase property items "reference name ". Property content is data object in SCD files in the format of LDName / LNName.FC.DO, where LDName = IEDName + LNInst; 2) 
Increase the protection signal type (Propnt), and discrete class format defined above.

Determine the mapping rules between IEC 61850 and IEC 61970 model as follows: 1) On the basis of 22 types of objects described in "CIM / E model describes the physical grid and Exchange Practices", coupled with the expansion of the protection signal, establish correspondence relationship with model defined in IEC 61850-6. As for the model not matched, refer to the existing type expansion; 2) From the substation class, desc model of the following examples in IEC 61850SCD corresponds to standard full path name (pathName) in IEC 61970CIM / E, which is named for the Chinese dispatching.

Do classification management for information. The information content comes from the relay protection information specification and monitoring system information specification, including remote measurement, remote signaling, remote control, remote investigation and protection information, which is export basis of the basic measurement.

The SCD file generated by the substation meets the specification of SCD engineering application management requirements. The typical requirements are that for each layer the object is in desc substation fill in the Chinese description. Layer by layer stitching can generate naming specification to meet the dispatching side, and the whole network only equipment or signal identification. It serves as a bridge between the master station data association map, mode and library. the English name IEC61850 model used, ID model substation used, the master model of information (or measurements) quoted by graphics files, and the use of IEC60870-5-104 protocol are linked by the number link common used.

In section SSD, through the private namespace, the expansion device (typical equipment for the bus, corresponding the bus in the IEC61970) and LNode attributes, relate the primary equipment directly to the data attribute of secondary signal, not by other rules or templates. It can be converted directly to test information for CIM/E in quantity. The basic principle of interval divided is the divided according to the functions of primary equipment. One interval contains only one main equipment or components with other devices connected, such as every bus is modeled as a independent interval, each circuit breaker builds a interval, and the transformer modeling for an independent interval and so on. At the same time, the model and graphics files are provided to dispatch center, and the model can be compared with each other.

On the basis of the substation and the dispatch center side of each system not changed, mapping and transformation from 61850 model to 61970 model is done simply and efficiently. It realizes model and information sharing, and Lays the foundation of the collaborative interaction to application function. It protects the original investment, and significantly reduces the maintenance workload of dispatch center model. It avoids duplication of work and improves work efficiency.

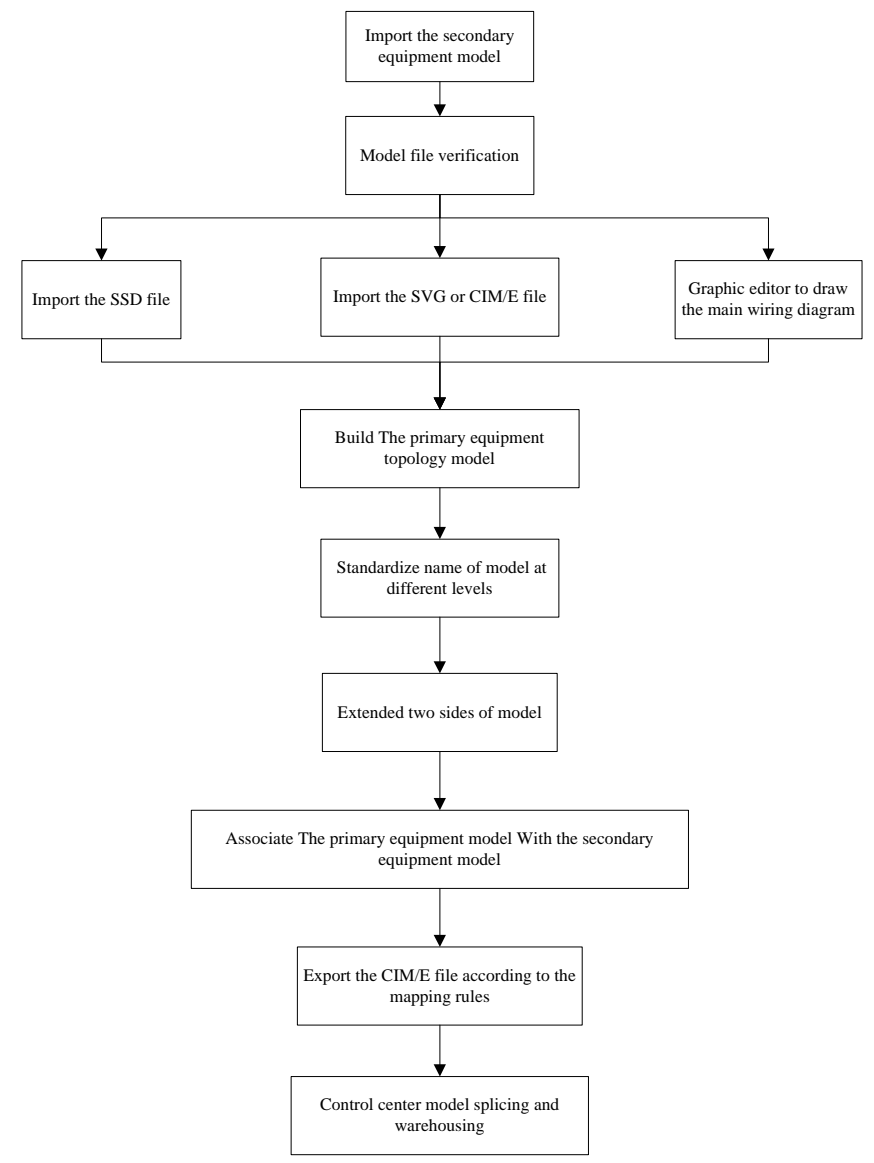

FIGURE II. INATION MODELING METHOD FOR SUBSTATION AND DISPATCH CENTER

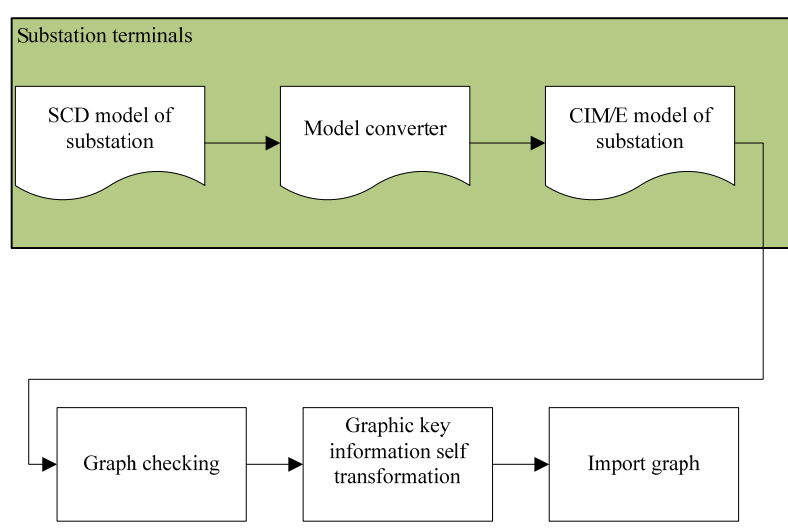

FIGURE III. GRAPHIC SHARING PROCESS 

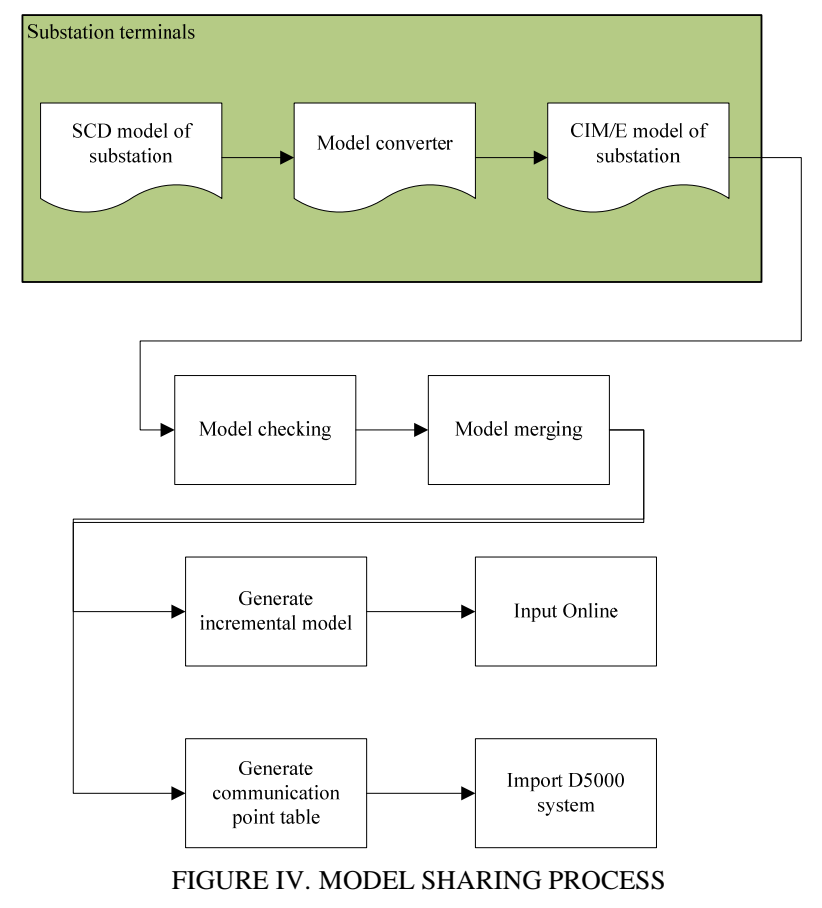

III. Modeling Process

\section{A. General Principle}

Integrated Modeling is done by offline model management center of the dispatch center smart grid dispatch technical support system. It's done primarily through model spliting or merging, version management and other modules. In this scenario, the integrated modeling merges dispatch center model with a single plant model, just considering the border between the dispatch center and one plant model. It is less the border and easier to maintain than the traditional model. But there may be problem that border information is not overlapping. To solve the problem, it's asked for detailed modeling for edge equipment, and smart boundary maintenance takes the method of force matching, based on boundary equipment of the station side, along with the boundary equipment of the dispatch center, giving access to the dispatch center model. The combined results are thrown into the offline database connectivity incrementally through the running system without disturbance into line after checked.

The substation system generates SCD model according to the configuration information provided by the system configuration module, and generates CIM/E and CIM/G files according to the mapping relationship between the 61850 model and 61970 model. Dispatch centre client is divided into online client and offline client. Online client realizes the communication between substation and dispatch centre based on protocol IEC 61850. Offline client uses the file service to receive $\mathrm{CIM} / \mathrm{E}$, $\mathrm{CIM} / \mathrm{G}$ files. After checking file, triggering the merge process model, the new information model is generated. After the completion of the merger, key information of CIM/G triggers self-conversion according to key information table generated during the merge process. At last, version management module releases the new information model to the subscriber, for the smart grid dispatching support system to provide the model / graphics.

Substation dispatching technical support system named standard visual modeling and rendering based on the realization of automatic data association modeling (generation master IEC61850 client communication required, to achieve the ICD model file) substation panoramic data source end maintenance.

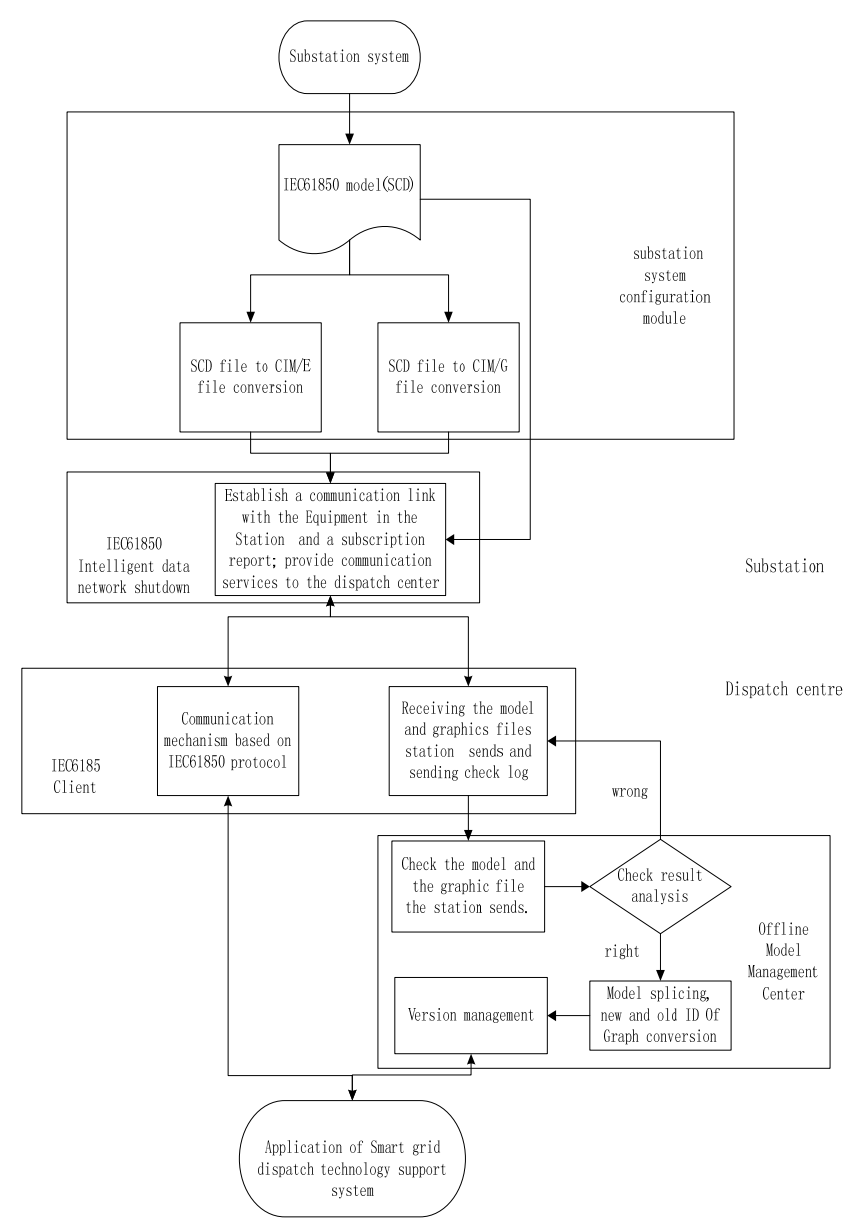

FIGURE V. INTEGRATED MODELING PROCESS OF SUBSTATION AND DISPATCH CENTER

\section{B. Modeling Scheme of Bay Level}

It is divided according to the principle of one main component in a space. In the 3/2 connection, angle wiring and wire change group connection mode, interval should be divided by circuit breakers, lines, short lead, main transformer, bus, high resistance and other main equipment. Dual bus wiring and other wiring methods shall be divided into the circuit breaker interval.

a) In $3 / 2$ connection and angle wiring way, the line and short lead interval are divided by switch.

b) each section of bus bar equipment should be created independently, including bus, bus voltage transformer, bus PT switch, rounding switch, etc... 
c) the transformer (including the main transformer and the transformer station used) should create independent intervals, that is, the transformer interval. Interval belongs to the voltage level of the transformer high voltage side winding. The transformer interval contains only one transformer equipment object (including the transformer body and the windings of the transformer). It should not contain other equipment objects, such as the connection of the circuit breaker, etc...

d) The secondary equipment related to the primary equipment, belongs to the interval of the primary equipment. Through the logical node, the interval of the primary equipment (main equipment or conductive equipment) establishes association.

e) The secondary equipment or system with no direct relation to the primary equipment, such as security fire control system, etc., should be set up the virtual interval according to the function, and associate through the logical node and the corresponding virtual interval. For the function of the total station public secondary equipment or system, the virtual interval is established at the highest voltage level, and others should be built in its function and the corresponding voltage level.

\section{MODELING EXAMPLE}

Taking the 35 Jia -9 switch interval as an example, the specific modeling examples of the expansion of bus type, the primary and secondary equipment functional logical nodes are given.

$<$ ConductingEquipment desc="35 Jia-9 switch" name="35J9" type="QF">

$<$ Private ext:dType="Normal Switch" type="CIME-type"/> $<$ LNode iedName="CM3501" ldInst="CTRL" lnClass="GGIO" lnInst="4" prefix="'>

<Private ext:dType=" The opening and closing state " ext:daName="stVal" ext:doName="Ind" ext:fc="ST" type="CIME-Discrete"/>

$</$ LNode $>$

$<$ Terminal bayName="CM3501" cNodeName= "CNode1154068-17" connectivityNode=" Testing/ 35kV.CM3501/CNode-1154068-17" substationName=" Testing station" voltageLevelName="35kV"/>

$<$ Terminal bayName="CM3501" cNodeName $=$ "CNode1154068-5" connectivityNode=" Testing/ 35kV.CM3501/CNode-1154068-5" substationName=" Testing station" voltageLevelName="35kV"/>

$</$ ConductingEquipment $>$

$<$ ConductingEquipment desc $=" 35 \mathrm{kV}-4$ Jia bus" name="35kV-4-jia" type="EBUS">

$<$ Private ext:dType="' type="CIME-type"/>

$<$ Terminal bayName="CM3501" cNodeName= "CNode1154068-83"

connectivityNode="Testing/35kV.CM3501/CNode-115406883" substationName="Testing station" voltageLevelName="35kV"/>

$</$ ConductingEquipment $>$
$<$ ConductingEquipment desc="35kV-5 Jia bus" name="35kV-5-jia" type="EBUS" $>$

$<$ Private ext:dType="' type="CIME-type"/>

$<$ Terminal bayName="CM3501" cNodeName= "CNode1154068-5" connectivityNode=" Testing /35kV.CM3501/CNode-1154068-5" substationName=" Testing station " voltageLevelName="35kV"/>

$</$ ConductingEquipment>

$<$ ConnectivityNode desc="CNode-1154068-5" name $=$ "CNode-1154068-5" pathName=" Testing /35kV.CM3501/ CNode-1154068-5"/>

$<$ ConnectivityNode desc="CNode-1154068-17" name $=$ "CNode-1154068-17" pathName=" Testing /35kV.CM3501/ CNode-1154068-17"/>

$<$ ConnectivityNode desc="CNode-1154068-83" name $=$ "CNode-1154068-83" pathName=" Testing /35kV.CM3501/ CNode-1154068-83"/>

$<$ ConnectivityNode desc="CNode-1154068-96" name $=$ "CNode-1154068-96" pathName=" Testing /35kV.CM3501/ CNode-1154068-96"/>

$<$ /Bay $>$

\section{CONCLUSION}

From the model depth integration of substation and dispatch center perspective, the article analyses modeling mode and technology of the substation and dispatch center, then gives the concrete implementation method of unified modeling and configuration in order to realize the information model of the source of the whole network sharing to provide practical solutions. It realizes source maintenance and coordination sharing of the dispatch center and substation model. It improves the file configuration detection and management standardization of substation, and establishes integrated operation control center and substation maintenance system to meet the needs of safe and stable operation of the grid.

\section{REFERENCES}

[1] Y.Jianguo, Y.Sheng, Y.Shengchun, Research on the Key Technology of Intelligent Dispatching[C]. International Conference on UHV power transmission technolog,2009.

[2] Haifeng, C.Yang, S.Xin,Power, Network Model Management System Fitted for Smart Dipatch[J]. Proceedings of the CSEE,2009,29(s1),pp:710.

[3] G.Zhiyuan, Y.Jianguo, C.Yang, Hierarchical Cyber-Threat Situation Evaluation Method for Electric Power Information Network[J]. Electric Power,2013,46(7),pp:116-120.

[4] Z.Haidong, Z.Hong, S.Xin, Model Conversion Method from Smart Substation SCD to Control Center CIM/E[J]. Automation of Electric Power Systems,2012,36(15),pp:91-95.

[5] G.Zhiyuan, Y.Jianguo, C.Yang, A Survey of Coordination Scheme Between CIM and IEC 61850 Model[J]. Automation of Electric Power Systems,2011,35(16),pp:9-14.

[6] H.Shaoqian, L.Li, Q.Zhong, Research on and Application of IEC 61850 Modelling and CIM Extension for Protection Relay Information Management System[J] .Automation of Electric Power Systems,2016,40(6),pp.119-125.

[7] W.Dongxia, S.Guangde, L.Wenbiao, Graphic conversion method for remote browser and source maintenance of smart substation[J].Power System Protection and Control,2014,42(18),pp.89-93. 
[8] D.Jun, H.Shaoqian, T.Jingyu, Application of data cource maintenance technology in relay protection information system[J].Electric Power Automation Equipment,2015,35(3),pp.162-169.

[9] H.Kai, Y.Ji, G.Quan, A Source-based Maintenance Technology for Integrated Intelligent Operation System of Power Grids [J].Automation of Electric Power Systems,2014,38(15)pp.71-75 